\title{
Patient-Reported Outcomes in Crohn's Disease: Does Race Matter?
}

\author{
Sara Horst
}

Received: 21 July 2014/ Accepted: 24 July 2014/Published online: 12 August 2014

(c) Springer Science+Business Media New York 2014

Inflammatory bowel disease (IBD), including Crohn's disease, has been recognized previously as a group of diseases primarily affecting Caucasians. Yet, recently reported data have supported the supposition that IBD is no longer a rarity in non-Caucasians, although still less common than in the Caucasian population [1, 2]. Also, inflammatory bowel disease may be increasing in incidence in certain US minority populations, including AfricanAmericans, according to hospital statistics [3-5].

Therefore, there is increasing interest in evaluation of the natural history of inflammatory bowel disease in the context of race and ethnicity. A systematic review published in 2013 addressed the racial, ethnic, and socioeconomic factors underlying IBD healthcare delivery and effectiveness, analyzing 42 studies, $88 \%$ retrospective, and primarily derived from the clinic setting (36\%) or from administrative or national survey data (33\%) [6]. Several possible race-based differences in healthcare delivery emerged from this analysis, including the observation that Caucasians were more often treated with immunomodulators and infliximab, suggesting a possible disparity in access to or the utilization of disease-modifying therapy. Nevertheless, a recently published study based on data from the National Ambulatory Medical Care Survey and the National Hospital Ambulatory Medical Care Survey reported no difference in medication use among Caucasians, African-American, Hispanic, or Asian patient populations [7]. In terms of IBD-related surgical outcomes,

\footnotetext{
S. Horst ( $\square)$

Department of Gastroenterology, Hepatology, and Nutrition, Inflammatory Bowel Disease Center, Vanderbilt University, 121121 st Ave, South, 220 Medical Arts Building, Nashville, TN 37232, USA

e-mail: sara.n.horst@vanderbilt.edu
}

two large studies evaluating a nationally representative hospitalization database have reported that African-American patients with Crohn's disease were significantly less likely to have undergone bowel resection [8,9].

Given that there are some indications of differences in health outcomes in Crohn's disease based on race, racedbased differences in health-related quality of life (HRQOL) or disease activity in patients with Crohn's disease may exist. In the above-mentioned systematic review, the few studies evaluating patient-reported disease activity confirmed that disease activity was generally similar in African-Americans and in Caucasians [6]. Only one prior case control study has reported the influence of race on HRQOL in patients with Crohn's disease [10]. In a Crohn's population, African-American patients were matched to Caucasian patients in order to evaluate several outcomes, including HRQOL. Patients were contacted via mail or telephone, with $\sim 60 \%$ of patients identified for the study, including 145 African-Americans and 407 Caucasians. Clinical factors, including number of hospitalizations, surgeries, and medication use were roughly equal in the two groups. There was no difference in HRQOL measures between African-American and Caucasian patients as measured by the Medical Outcomes Short Form 36 (SF-36) after adjusting for age, gender, study site, educational level, and disease severity.

In this issue of Digestive Diseases and Sciences, Ghazi et al. [11] reported no differences in HRQOL and disease activity measures between African-American and Caucasians. In the study, 296 patients with Crohn's disease (19\% African-American) treated at a single tertiary care medical center specialty inflammatory bowel disease clinic underwent HRQOL evaluation using the Short Form Inflammatory Bowel Disease Questionnaire (SIBDQ) with disease activity measured by the Harvey-Bradshaw Index (HBI). 
Data collected from patients evaluated at routine clinic visits were retrospectively reviewed and, when possible, evaluated over multiple time points as the patients returned for subsequent clinic visits. African-American and Caucasian patient cohorts were similar, with no significant differences in age, gender, smoking status, disease phenotype, or medication exposure at baseline. No statistical differences were reported between the groups for stricturing, penetrating, or perianal disease, with similar baseline HRQOL scores, with significant improvements over baseline but not between groups during follow-up. Baseline disease activity scores were also similar between groups, with scores decreasing significantly in the first 180 day follow-up in the Caucasian patient population only. African-American patients had improvement in disease activity scores but did not reach statistical significance in the first 180 days of treatment. Disease activity scores remained relatively stable in the subsequent time frames and were not different between races. Again, the amount the disease activity score decreased was not statistically significantly different between African-American or Caucasian patients at any time point.

A strength is that the evaluation included a large proportion of African-American patients from a single center. Previously published studies have evaluated highly varying numbers of minority patients, which can significantly limit the ability to effectively assess outcomes [6]. Also, the advantage of restriction to a single center is that these patients likely received comparable care for their inflammatory bowel disease, as evidenced by a similar proportion of patients receiving each type of immunosuppressive medications. Still, further support of this apparent trend can always be strengthened by including multiple centers in further evaluation and enrolling patients in a prospective study to limit loss of follow-up data. Indeed, in this retrospective analysis, the number of patients with available data in each 180-day window decreased over time (only 84 patients in the last data collection window approximately 3 years after an initial visit).

In the study, disease activity was evaluated using the Harvey-Bradshaw Index, a well-validated and widely used measure for evaluating Crohn's disease activity. In recent years, this and other measures have been under scrutiny for lack of correlation to objective markers of disease activity such as ileocolonoscopy [12]. Yet, it is important that this tool which compiles patient-reported symptoms and experiences (well-being, stool frequency, and abdominal pain) and physical examination findings was not different between African-American and Caucasian patients over time.

The ability to evaluate disease activity and HRQOL data over time in the study in most of the patient cohort $(90 \%$ had a second data point the second data collection point, at approximately 180 days) instead of a "snapshot" lessens the likelihood of the result being secondary to chance alone. Also, these two populations were similar for most other covariates, including age, gender, smoking status, disease phenotype, and medication exposure, allowing that if race or ethnicity could have affected the outcome of disease activity, it would have likely been evident. Therefore, this study adds to the understanding of possible racial disparities, or in this case, the lack thereof, when evaluating patient outcomes in patients with inflammatory bowel disease. It is reassuring that HRQOL and disease activity were similar in an African-American and Caucasian population with similar demographics and disease phenotype. Importantly, this study underscores the importance of consistency of medical care regardless of ethnicity or race, as outcomes over time were not different and the percentage of patients on differing immunosuppressive therapies were similar between the African-American and Caucasian patients. Therefore, treatment of inflammatory bowel disease should continue to focus on tailoring therapy to disease severity. Appropriate therapies such as anti-TNF therapies and other biologic medications and immunomodulators can and should be instituted regardless of race or ethnicity. Future studies with prospective and multicenter evaluation are likely indicated, which might also focus on other factors that may inform HRQOL such as socioeconomic status.

\section{References}

1. Wang YR, Loftus EV Jr, Cangemi JR, et al. Racial/ethnic and regional differences in the prevalence of inflammatory bowel disease in the United States. Digestion. 2013;88:20-25.

2. Kurata JH, Kantor-Fish S, Frankl H, et al. Crohn's disease among ethnic groups in a large health maintenance organization. Gastroenterology. 1992;102:1940-1948.

3. Sewell JL, Yee HF Jr, Inadomi JM. Hospitalizations are increasing among minority patients with Crohn's disease and ulcerative colitis. Inflamm Bowel Dis. 2010;16:204-207.

4. Basu D, Lopez I, Kulkarni A, et al. Impact of race and ethnicity on inflammatory bowel disease. Am J Gastroenterol. 2005;100: 2254-2261.

5. Cross RK, Jung C, Wasan S, et al. Racial differences in disease phenotypes in patients with Crohn's disease. Inflamm Bowel Dis. 2006;12:192-198.

6. Sewell JL, Velayos FS. Systematic review: the role of race and socioeconomic factors on IBD healthcare delivery and effectiveness. Inflamm Bowel Dis. 2013;19:627-643.

7. Lin KK, Sewell JL. The effects of race and socioeconomic status on immunomodulator and anti-tumor necrosis factor use among ambulatory patients with inflammatory bowel disease in the United States. Am J Gastroenterol. 2013;108:1824-1830.

8. Nguyen GC, Bayless TM, Powe NR, et al. Race and health insurance are predictors of hospitalized Crohn's disease patients undergoing bowel resection. Inflamm Bowel Dis. 2007;13: 1408-1416. 
9. Nguyen GC, Laveist TA, Gearhart S, et al. Racial and geographic variations in colectomy rates among hospitalized ulcerative colitis patients. Clin Gastroenterol Hepatol. 2006;4: 1507-1513.

10. Straus WL, Eisen GM, Sandler RS, et al. Crohn's disease: does race matter? The Mid-Atlantic Crohn's Disease Study Group. Am J Gastroenterol. 2000;95:479-483.
11. Ghazi LJ, Lydecker AD, Patil SA, et al. Racial Differences in disease activity and quality of life in patients with Crohn's disease. Dig Dis Sci. (Epub ahead of print). doi:10.1007/s10620014-3141-3.

12. Peyrin-Biroulet L, Reinisch W, Colombel JF, et al. Clinical disease activity, C-reactive protein normalisation and mucosal healing in Crohn's disease in the SONIC trial. Gut. 2014;63:88-95. 